\title{
Association of pre-percutaneous coronary flow grade and clinical outcomes in patients with non-ST-segment elevation myocardial infarction Data from the Korea Acute Myocardial Infarction Registry
}

\author{
Yong Hoon Kim, MD, PhDa,* (D) Ae-Young Her, MD, PhDª, Myung Ho Jeong, MD, PhD ${ }^{\mathrm{b}}$, \\ Byeong-Keuk Kim, MD, $\mathrm{PhD}^{\mathrm{c}}$, Sung-Jin Hong, $\mathrm{MD}^{\mathrm{c}}$, Seunghwan Kim, MD ${ }^{\mathrm{d}}$, Chul-Min Ahn, MD, $\mathrm{PhD}^{\mathrm{c}}$, \\ Jung-Sun Kim, MD, $\mathrm{PhD}^{\mathrm{C}}$, Young-Guk Ko, MD, $\mathrm{PhD}^{\mathrm{C}}$, Donghoon Choi, $\mathrm{MD}, \mathrm{PhD}^{\mathrm{C}}$, \\ Myeong-Ki Hong, MD, $\mathrm{PhD}^{\mathrm{c}}$, Yangsoo Jang, $\mathrm{MD}, \mathrm{PhD}^{\mathrm{C}}$
}

\begin{abstract}
Because of a paucity of published data, we compared the 2-year major clinical outcomes between pre-percutaneous coronary intervention (pre-PCl) thrombolysis in myocardial infarction (TIMI) flow grade 0/1 (pre-TIMI flow grade [pre-TIMI] 0/1) group and pre$\mathrm{PCl}$ TIMI flow grade 2/3 (pre-TIMI 2/3) group in patients with non-ST-segment elevation myocardial infarction (NSTEMI) who underwent successful implantation of newer-generation drug-eluting stent.

A total of 7506 NSTEMI patients were divided into 2 groups: pre-TIMI 0/1 group ( $n=3157$ ) and pre-TIMI 2/3 group ( $n=4349$ ). The primary outcome was major adverse cardiac events defined as all-cause death, recurrent myocardial infarction, or any repeat revascularization. The secondary outcome was stent thrombosis (ST).

After propensity score-matched (PSM) analysis, 2 PSM groups (2473 pairs, $n=4946$, C-statistic=0.684) were generated. Major adverse cardiac events (hazard ration [HR], 1.294; 95\% confidence interval [Cl]: 1.065-1.572; $P=.009$ ), all-cause death (HR, 1.559, $P=.003)$, cardiac death (HR: 1.641, $P=.005)$, and all-cause death or $\mathrm{Ml}(\mathrm{HR}: 1.531, P=.001)$ rates were significantly higher in the pre-TIMI 0/1 group than in the pre-TIMI 2/3 group. Moreover, these differences were more prominent during the first 1 month after the index PCl. However, the cumulative incidences of recurrent myocardial infarction, any revascularization, and ST were similar between the 2 groups.

Among a contemporary cohort of NSTEMI, these data suggest that the presence of a pre-PCl patency of the infarct-related artery showed better mortality reduction capacity than those with a lack of patency.

Abbreviations: DES = drug-eluting stent, KAMIR = Korea Acute Myocardial Infarction Registry, MACEs = major adverse cardiac events, NSTEMI = non-ST-elevation myocardial infarction, $\mathrm{PCl}=$ percutaneous coronary intervention, Pre-TIMI = pre-TIMI flow grade, $\mathrm{Re}-\mathrm{Ml}=$ recurrent myocardial infarction, $\mathrm{ST}=$ stent thrombosis, TIMI = thrombolysis in myocardial infarction.
\end{abstract}

Keywords: non-stent thrombosis-segment elevation myocardial infarction, percutaneous coronary intervention, reperfusion

Editor: Flavio Palmieri

YHK and A-YH contributed equally to this work.

This research was supported by a fund (2016-ER6304-02) by Research of Korea Centers for Disease Control and Prevention.

The authors have no conflicts of interest to disclose.

Supplemental Digital Content is available for this article.

All data generated or analyzed during this study are included in this published article [and its supplementary information files].

${ }^{a}$ Division of Cardiology, Department of Internal Medicine, Kangwon National University School of Medicine, Chuncheon, Republic of Korea, ${ }^{b}$ Cardiovascular Center, Department of Cardiology, Chonnam National University Hospital, Gwangju, Republic of Korea, ' Division of Cardiology, Severance Cardiovascular Hospital, Yonsei University College of Medicine, Seoul, Republic of Korea, ${ }^{d}$ Division of Cardiology, Inje University College of Medicine, Haeundae Paik Hospital, Busan, Republic of Korea.

* Correspondence: Yong Hoon Kim, Division of Cardiology, Department of Internal Medicine, Kangwon National University School of Medicine 24289,156 Baengnyeong Road, Chuncheon City, Gangwon Province, Republic of Korea (e-mail: yhkim02@kangwon.ac.kr),

Copyright () 2021 the Author(s). Published by Wolters Kluwer Health, Inc.

This is an open access article distributed under the terms of the Creative Commons Attribution-Non Commercial License 4.0 (CCBY-NC), where it is permissible to download, share, remix, transform, and buildup the work provided it is properly cited. The work cannot be used commercially without permission from the journal.

How to cite this article: Kim YH, Her AY, Jeong MH, Kim BK, Hong SJ, Kim S, Ahn CM, Kim JS, Ko YG, Choi D, Hong MK, Jang Y. Association of pre-percutaneous coronary flow grade and clinical outcomes in patients with non-ST-segment elevation myocardial infarction: Data from the Korea Acute Myocardial Infarction Registry. Medicine 2021;100:32(e26947).

Received: 5 January 2021 / Received in final form: 14 June 2021 / Accepted: 15 July 2021

http://dx.doi.org/10.1097/MD.0000000000026947 


\section{Introduction}

The presence or absence of reperfusion of an infarct-related artery (IRA) is a major determinant of prognosis in patients with acute myocardial infarction (AMI). ${ }^{[1-3]}$ In the harmonizing outcomes with revascularization and stents in acute myocardial infarction (HORIZONS-AMI) trial, ${ }^{[4]}$ the presence of early IRA patency, defined as thrombolysis in myocardial infarction (TIMI) flow grade $2 / 3$, was associated with lower rates of 1 -year mortality $(2.5 \%$ vs $3.9 \%, P=.04)$ than those with TIMI flow grade $0 / 1$. Similarly, Stone et a ${ }^{[3]}$ showed that pre-percutaneous coronary intervention (pre-PCI) TIMI flow grade 3 (pre-TIMI 3) was an independent predictor of survival in patients with STsegment elevation myocardial infarction (STEMI). However, in the case of non-STEMI (NSTEMI), the published comparative data regarding the long-term effects of the patency of pre-PCI IRA were very limited. In a study by Bailleul et al,${ }^{[5]}$ the risk of death after adjustment was similar between the pre-TIMI $0 / 1$ and pre-TIMI 2/3 groups during a 3-year follow-up period (hazard ratio [HR]: $0.79 ; 95 \%$ confidence interval [CI]: 0.56-1.11; $P=.17)$. De Luca et $\mathrm{al}^{[6]}$ reported that reduced pre-PCI TIMI flow in patients with acute coronary syndrome (ACS) did not affect the survival at 1 year. Theoretical advantages of early IRA patency lead to easier guidewire passage and a smaller thrombus burden with a lower risk of distal embolization. ${ }^{[4]}$ Other advantages include decreased enzymatic infarct size, fatal arrhythmic events, and in-hospital mortality rate. ${ }^{[7]}$ In a contemporary newergeneration drug-eluting stent (DES) era, to provide more clear information concerning the effects of patency of pre-PCI IRA in patients with NSTEMI during the long-term follow-up period, we investigated the 2-year major clinical outcomes of these 2 groups based on "lack of patency" (pre-TIMI 0/1) or "patency" (preTIMI 2/3) status ${ }^{[8]}$ in patients with NSTEMI who underwent successful implantation of newer-generation drug-eluting stent (DES).

\section{Methods}

\subsection{Study population}

Overall, 9615 patients with NSTEMI underwent successful PCI using newer-generation DESs from November 2005 to June 2015 in the Korea acute myocardial infarction registry (KAMIR) ${ }^{[9]}$ were evaluated. The KAMIR is a nationwide prospective multicenter registry in South Korea that was established in November 2005. The details of this registry can be found at the KAMIR website (http://www.kamir.or.kr). Eligible patients were aged $\geq 18$ years at the time of hospital admission. Among them, those with incomplete laboratory results $(\mathrm{n}=1728,18.0 \%)$ and who were lost to follow-up ( $\mathrm{n}=381,3.9 \%$ ) were excluded. Finally, a total of 7506 NSTEMI patients were included. These patients were divided into 2 groups: pre-TIMI 0/1 group $(\mathrm{n}=3157,42.1 \%)$ and preTIMI $2 / 3$ group $(n=4349,57.9 \%$ ) (Fig. 1). All patients provided written informed consent before enrollment. The follow-up data were collected through face-to-face interviews, phone calls, and medical records reviews. Altogether, 7506 NSTEMI patients completed the scheduled follow-up. Additionally, an independent event-adjudicating committee evaluated all clinical events. The processes of the event adjudication process have been described in a previous publication by the KAMIR investigators. ${ }^{[9]}$ This study protocol was approved by the ethics committee at each participating center and the Chonnam National University Hospital Institutional Review Board (IRB) ethics committee (CNUH-2011-172) according to the ethical guidelines of the 1975 Declaration of Helsinki.

\subsection{Percutaneous coronary intervention and medical treatments}

Coronary angiography and PCI was performed in accordance with relevant standard techniques at the time of each

Overall, 9615 patients with NSTEMI who underwent successful PCI using newer-generation DESs from November 2005 to June 2015 in the KAMIR were evaluated

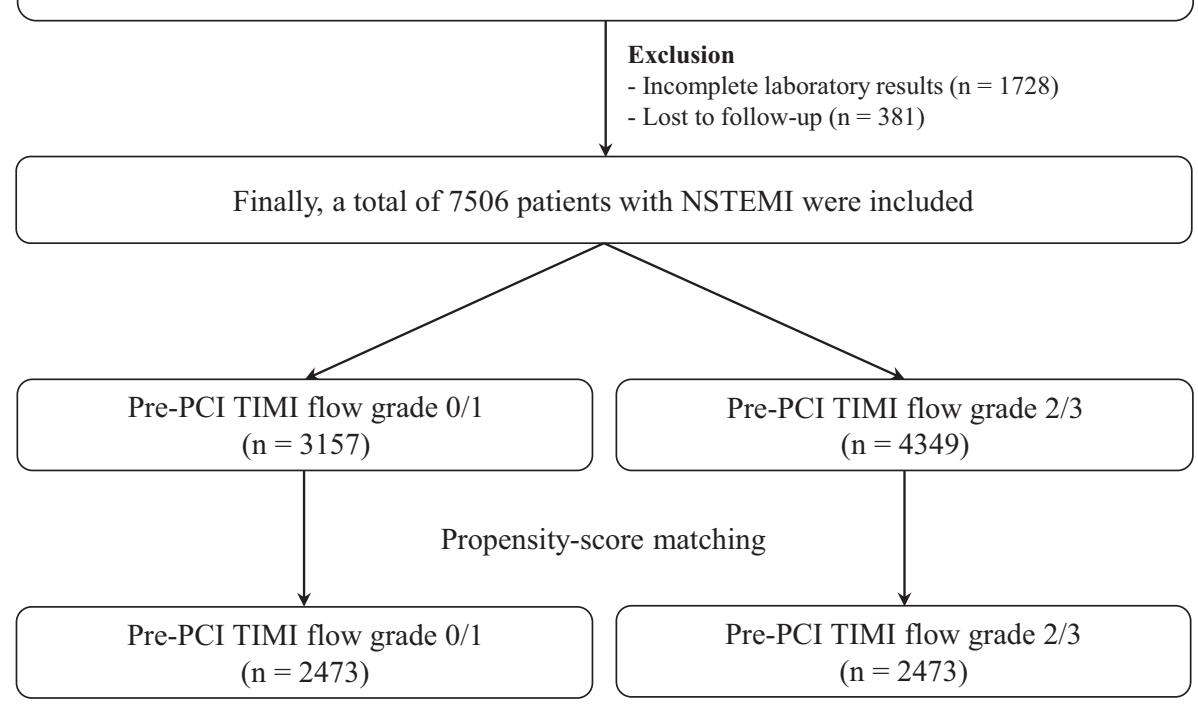

Figure 1. Flow chart. 
procedure $^{[10]}$ after the intravenous infusion of unfractionated heparin $(50-100 \mathrm{U} / \mathrm{kg})$ to achieve an activated clotting time of $>250$ seconds. Before PCI, all patients received loading doses of aspirin 200 to $300 \mathrm{mg}$ and clopidogrel 300 to $600 \mathrm{mg}$, or ticagrelor $180 \mathrm{mg}$ or prasugrel $60 \mathrm{mg}$ was administered. The total duration of recommended dual antiplatelet therapy (DAPT; a combination of aspirin $100 \mathrm{mg} / \mathrm{d}$ and clopidogrel $75 \mathrm{mg} / \mathrm{d}$ or ticagrelor $90 \mathrm{mg}$ twice daily or prasugrel $5-10 \mathrm{mg} / \mathrm{d}$ ) was $\geq 12$ months. Moreover, the access site, revascularization strategy, and selection of DES were left to the discretion of the individual operators.

\subsection{Study definitions and endpoints}

NSTEMI was defined according to the current guidelines. ${ }^{[11,12]}$ Before PCI, the degree of coronary flow was classified according to TIMI flow grade, as assessed by the investigators. ${ }^{[13]}$ Successful PCI was defined as $<30 \%$ residual stenosis and a TIMI flow grade 3 for the IRA after the procedure. The primary clinical outcome of this study was the occurrence of major adverse cardiac events (MACEs) defined as all-cause death, recurrent myocardial infarction (re-MI), or any coronary repeat revascularization. The secondary outcome was definite or probable stent thrombosis (ST) during a 2-year follow-up period. All-cause death was classified as cardiac death $(\mathrm{CD})$ or non-CD. Any repeat revascularization included target lesion revascularization (TLR), target vessel revascularization (TVR), and non-TVR. The definitions of re-MI, TLR, TVR, and non-TVR are as previously reported. ${ }^{[14]}$

\subsection{Statistical analysis}

Categorical data are reported as numbers and percentages and were compared using chi-square or Fisher's exact test as appropriate. Continuous variables are expressed as mean \pm standard deviation and compared with the Student's $t$ test. The propensity score-matched (PSM) analysis was performed using a logistic regression model to adjust for potential confounders. We tested all available variables that could be of potential relevance, such as all baseline clinical, angiographic, and procedural factors as shown in Tables 1 and 2. The C-statistics for PSM was 0.684 in this study. Patients in the pre-TIMI 0/1 group were then one-to-one matched to those in the pre-TIMI $2 / 3$ group according to propensity scores with the nearest available pair matching method. For this PSM, a one-to-one matching process without replacement was performed by a greedy algorithm with a caliper width 0.01 standard deviations. The procedure yielded 2564 matched pairs (Fig. 1). Cox-proportional hazard models were used to assess the adjusted HR comparing the 2 groups in the PSM population. Various clinical outcomes were estimated using the Kaplan-Meier curve analysis, and the intergroup difference was compared using the log-rank test. For all analyses, a 2 -sided $P<.05$ was considered statistically significant. All statistical analyses were performed using SPSS software version 20 (IBM, Armonk, NY).

\section{Results}

\subsection{Baseline characteristics}

Tables 1 and 2 show the baseline characteristics of the study population. In the total study population, the number of men was higher in the pre-TIMI 0/1 group than in the pre-TIMI $2 / 3$ group $(72.7 \%$ vs $70.2 \%, P=.020)$. The mean age $(65.3 \pm 11.7$ years vs
$63.3 \pm 12.2$ years, $P<.001)$ and mean left ventricular ejection fraction (LVEF, $55.0 \pm 11.1 \%$ vs $53.3 \pm 11.0 \%, P<.001$ ) of the pre-TIMI $2 / 3$ group were higher than the pre-TIMI 0/1 group. The total number of current smokers; mean peak blood levels of creatine kinase myocardial band (CK-MB), troponin-I; mean high-sensitivity C-reactive protein; mean total cholesterol, triglyceride, high-density lipoprotein-cholesterol, and low-density lipoprotein-cholesterol; the prescription rate of clopidogrel as a discharge medication; the numbers of left circumflex coronary artery and right coronary artery as the IRA and treated vessels; American College of Cardiology/American Heart Association (ACC/AHA) type C lesion: in-hospital use of glycoprotein (GP) IIb/IIIa inhibitor; PCI within 24 hours; and mean length of deployed stents were significantly higher in the pre-TIMI $0 / 1$ group than in the pre-TIMI $2 / 3$ group. In contrast, the number of patients who had history of hypertension, diabetes mellitus (DM), dyslipidemia, and PCI; the mean value of $\mathrm{N}$-terminal probrain natriuretic peptide (NT-ProBNP); prescription rates of aspirin, ticagrelor, beta-blockers, angiotensin receptor blockers, calcium channel blockers, and lipid-lowering agents; left main coronary artery and left anterior descending coronary artery as the IRA and treated vessel; ACC/AHA type B2 lesion; uses of intravascular ultrasound; and mean deployed stent diameter were significantly higher in the pre-TIMI $2 / 3$ group than in the preTIMI 0/1 group. However, number of patients with cardiogenic shock, cardiopulmonary resuscitation (CRP) on admission, extent of coronary artery disease (single-vessel, 2-vessel, or 3 -vessel disease), and multivessel PCI were similar between the 2 groups. After PSM analysis, these baseline differences between the 2 groups were well balanced.

\subsection{Clinical outcomes}

Table 3 shows the clinical outcomes at 1 month and 2 years. Figure 2 shows Kaplan-Meier analysis for major clinical outcomes in the total study population and PSM patients at 2 years. During the first 1 month after index PCI, in total study population, the cumulative incidences of MACEs, all-cause death, CD, and all-cause death or MI were significantly higher in the pre-TIMI 0/1 group than in the pre-TIMI 2/3 group. After PSM analysis, the cumulative incidences of MACEs (HR, 1.519; 95\% CI: 1.042-2.214; $P=.030$ ), all-cause death (HR: 1.759; 95\% CI: 1.106-2.798; $P=.017$ ), CD (HR: 1.889; 95\% CI: $1.163-3.069 ; P=.010)$, and all-cause death or MI (HR: 1.641; 95\% CI: $1.096-2.457 ; P=.016)$ were also higher in the pre-TIMI $0 / 1$ group than in the pre-TIMI $2 / 3$ group. At 2 years, in the total study population, the cumulative incidences of MACEs, all-cause death, CD, and all-cause death or MI were significantly higher in the pre-TIMI 0/1 group than in the pre-TIMI 2/3 group. However, the cumulative incidences of re-MI, any repeat revascularization, and ST were not significantly different between the 2 groups. After PSM analysis, the cumulative incidence of MACEs (HR: 1.294; 95\% CI: 1.065-1.572; $P=.009$ ), all-cause death (HR, 1.559; 95\% CI, 1.165-2.087; $P=.003)$, CD (HR: 1.641; 95\% CI: $1.159-2.325 ; P=.005)$, and all-cause death or MI (HR: 1.531; 95\% CI: 1.196-1.960; $P=.001$ ) were significantly higher in the pre-TIMI $0 / 1$ group than in the preTIMI 2/3 group. However, the cumulative incidence of re-MI (HR: $1.393 ; 95 \%$ CI: 0.914-2.124; $P=.123$ ), any repeat revascularization (HR: 1.054; 95\% CI, 0.789-1.408; $P=.723$ ), and ST (HR: $1.302 ; 95 \%$ CI: 0.571-2.969; $P=.530$ ) were similar between the 2 groups. Figure S1, Supplemental Digital Content, 
Table 1

Baseline clinical and laboratory characteristics and discharge medication.

\begin{tabular}{|c|c|c|c|c|c|c|c|c|}
\hline \multirow[b]{2}{*}{ Variables } & \multicolumn{4}{|c|}{ Total study population $(n=7506)$} & \multicolumn{4}{|c|}{ PSM patients $(n=4946)$} \\
\hline & $\begin{array}{c}\text { Pre-PCI TIMI 0/1 } \\
(n=3157)\end{array}$ & $\begin{array}{c}\text { Pre-PCI TIMI } 2 / 3 \\
(n=4349)\end{array}$ & $P$ value & SD & $\begin{array}{c}\text { Pre-PCI TIMI 0/1 } \\
(n=2473)\end{array}$ & $\begin{array}{l}\text { Pre-PCI TIMI } 2 / 3 \\
(n=2473)\end{array}$ & $P$ value & SD \\
\hline Age, yrs & $63.3 \pm 12.2$ & $65.3 \pm 11.7$ & $<.001$ & -1.67 & $64.5 \pm 11.7$ & $64.4 \pm 12.0$ & .729 & 0.08 \\
\hline$\geq 65$ years, $n(\%)$ & $1471(46.6)$ & 2341 (53.8) & $<.001$ & -1.91 & $1240(50.1)$ & $1247(50.4)$ & .842 & -0.08 \\
\hline LVEF, $\%$ & $53.3 \pm 11.0$ & $55.0 \pm 11.1$ & $<.001$ & -1.54 & $54.0 \pm 10.9$ & $53.8 \pm 10.9$ & .710 & 0.18 \\
\hline$<40 \%, n(\%)$ & $314(9.9)$ & $411(9.5)$ & .473 & 0.16 & $238(9.6)$ & $249(10.1)$ & .600 & -0.20 \\
\hline $\mathrm{DBP}, \mathrm{mmHg}$ & $80.5 \pm 15.7$ & $81.2 \pm 14.8$ & .042 & -0.46 & $80.8 \pm 15.4$ & $80.7 \pm 15.1$ & .760 & 0.07 \\
\hline Cardiogenic shock, n (\%) & $119(3.8)$ & $133(3.1)$ & .092 & 0.37 & $83(3.4)$ & $86(3.5)$ & .876 & -0.05 \\
\hline CPR on admission, $n(\%)$ & $79(2.5)$ & $106(2.4)$ & .880 & 0.06 & $59(2.4)$ & $54(2.2)$ & .704 & 0.11 \\
\hline Hypertension, $\mathrm{n}(\%)$ & $1552(49.2)$ & $2428(55.8)$ & $<.001$ & -1.74 & $1291(52.2)$ & $1290(52.2)$ & .977 & 0.01 \\
\hline Diabetes mellitus, n (\%) & $861(27.3)$ & $1418(32.6)$ & $<.001$ & -1.54 & $723(29.2)$ & $710(28.7)$ & .707 & 0.14 \\
\hline Dyslipidemia, n (\%) & $329(10.7)$ & $587(13.5)$ & $<.001$ & -1.09 & $285(11.5)$ & $291(11.8)$ & .825 & -0.11 \\
\hline Previous HF, n (\%) & $36(1.1)$ & $72(1.7)$ & .077 & -0.39 & $33(1.3)$ & $37(1.5)$ & .718 & -0.13 \\
\hline Current smokers, n (\%) & $1243(39.4)$ & 1574 (36.2) & .005 & 0.86 & 917 (37.1) & $934(37.8)$ & .638 & -0.19 \\
\hline Peak CK-MB, mg/dL & $84.8 \pm 118.7$ & $50.8 \pm 180.0$ & $<.001$ & 2.23 & $71.3 \pm 103.8$ & $64.5 \pm 232.9$ & .186 & 0.38 \\
\hline Peak Troponin-I, ng/mL & $29.6 \pm 92.7$ & $16.6 \pm 43.7$ & $<.001$ & 1.79 & $25.4 \pm 94.5$ & $23.7 \pm 53.5$ & .403 & 0.22 \\
\hline Blood glucose, mg/dL & $160.0 \pm 80.0$ & $160.3 \pm 80.5$ & .732 & -0.04 & $159.2 \pm 76.2$ & $159.0 \pm 77.9$ & .916 & 0.03 \\
\hline NT-ProBNP, pg/mL & $2399.4 \pm 3829.5$ & $2806.7 \pm 5995.0$ & $<.001$ & -0.81 & $2506.4 \pm 4109.5$ & $2540.2 \pm 4633.7$ & .786 & -0.08 \\
\hline Hs-sensitivity CRP, mg/dL & $9.21 \pm 37.6$ & $8.90 \pm 46.7$ & .179 & 0.07 & $8.9 \pm 36.1$ & $8.8 \pm 56.6$ & .931 & 0.02 \\
\hline Serum creatinine, mg/L & $1.11 \pm 1.22$ & $1.16 \pm 1.30$ & .075 & -0.40 & $1.1 \pm 1.3$ & $1.1 \pm 1.2$ & .877 & -0.04 \\
\hline Total cholesterol, mg/dL & $185.3 \pm 44.8$ & $178.2 \pm 46.8$ & $<.001$ & 1.55 & $183.1 \pm 44.1$ & $183.1 \pm 48.6$ & .973 & 0.01 \\
\hline Triglyceride, mg/L & $139.0 \pm 123.1$ & $132.3 \pm 108.4$ & .016 & 0.58 & $132.6 \pm 101.0$ & $136.0 \pm 115.2$ & .280 & -0.31 \\
\hline HDL cholesterol, mg/L & $43.4 \pm 16.0$ & $42.6 \pm 13.0$ & .030 & 0.55 & $43.1 \pm 12.9$ & $43.3 \pm 14.2$ & .586 & -0.15 \\
\hline LDL cholesterol, mg/L & $117.2 \pm 43.2$ & $112.4 \pm 36.9$ & $<.001$ & 1.19 & $116.1 \pm 44.2$ & $116.3 \pm 37.2$ & .891 & -0.05 \\
\hline ARBs, $n(\%)$ & $931(29.5)$ & $1486(34.2)$ & $<.001$ & -1.31 & $769(31.1)$ & $783(31.7)$ & .690 & -0.17 \\
\hline CCBs, $n(\%)$ & $221(7.0)$ & $448(10.3)$ & $<.001$ & -1.47 & $198(8.0)$ & $189(7.6)$ & .672 & 0.17 \\
\hline Lipid-lowering agents, n (\%) & $2736(86.7)$ & 3910 (89.9) & $<.001$ & -1.16 & $2176(88.0)$ & 2179 (88.1) & .895 & -0.04 \\
\hline
\end{tabular}

Values are means \pm SD or numbers and percentages. The $P$ values for continuous data were obtained from the unpaired $t$ test. The $P$ values for categorical data from chi-square or Fisher exact test. ACEls $=$ angiotensin converting enzyme inhibitors, $\mathrm{ARBs}=$ angiotensin receptor blockers, $\mathrm{BBs}=\mathrm{B}$-blockers, $\mathrm{BMI}=$ body mass index, $\mathrm{CABG}=\mathrm{coronary}$ artery bypass graft, $\mathrm{CCBS}=\mathrm{calcium}$ channel blockers, $\mathrm{CK}$-MB $=$ creatine kinase myocardial band, $\mathrm{CVA}=$ cerebrovascular accident, $\mathrm{DBP}=$ diastolic blood pressure, eGFR=estimated glomerular filtration rate, $\mathrm{HDL}=$ high-density lipoprotein, $\mathrm{HF}=$ heart failure, $\mathrm{Hs}$ - $\mathrm{CRP}=$ high sensitivity C-reactive protein, $\mathrm{LDL}=$ low-density lipoprotein, $\mathrm{LVEF}=$ left ventricular ejection fraction, $\mathrm{Ml}=$ myocardial infarction, $\mathrm{NT}$-ProBNP $=\mathrm{N}$-terminal pro-brain natriuretic peptide, $\mathrm{PCl}=$ percutaneous coronary intervention, Pre-PCl= pre-percutaneous coronary intervention, $\mathrm{PSM}=$ propensity-score matched, $\mathrm{SBP}=$ systolic blood pressure, $\mathrm{SD}=$ standardized mean difference, TIMI = thrombolysis in myocardial infarction.

http://links.lww.com/MD/G363 shows subgroup analysis for MACEs. Patients who did not have cardio genic shock, pre-TIMI 2/3 shows more beneficial effect on MACEs (HR: 1.18; 95\% CI: 1.01-1.39; $P=.035)$ in patients with NSTEMI compared with pre-TIMI $0 / 1$. However, HR for patients with cardiogenic shock was 1.55 , which was numerically larger than $H R$ for patients without cardiogenic shock. Hence, although it was not statistically significant due to small sample size, pre TIMI 2/3 may be associated with favorable outcomes in patients with cardiogenic shock. Table S1, Supplemental Digital Content, http://links.lww.com/MD/G364 shows independent predictors of all-cause death and cardiac death in PSM patients. Old age $(\geq 65$ years), hypertension, DM, previous PCI, decreased LVEF
$(<40 \%)$, cardiogenic shock, CPR on admission, angiotensinconverting enzyme inhibitor, lipid-lowering agent, multivessel disease, and the occurrence of ventricular tachycardia (VT)/ ventricular fibrillation (VF) were independent predictors of both all-cause death and CD.

\section{Discussion}

The main findings were as follows: first, both during the first 1 month and at 2 years, after PSM analysis, the cumulative incidence of MACEs, all-cause death, CD, and all-cause death or MI were significantly higher in the pre-TIMI 0/1 group than in the pre-TIMI 2/3 group. Second, both during the first 1 month and at 
Table 2

Angiographic and procedural characteristics.

\begin{tabular}{|c|c|c|c|c|c|c|c|c|}
\hline \multirow[b]{2}{*}{ Variables } & \multicolumn{4}{|c|}{ Total study population $(n=7506)$} & \multicolumn{4}{|c|}{ PSM patient $(n=4946)$} \\
\hline & $\begin{array}{l}\text { Pre-PCI TIMI 0/1 } \\
(\mathbf{n}=\mathbf{3 1 5 7 )}\end{array}$ & $\begin{array}{l}\text { Pre-PCI TIMI 2/3 } \\
(n=4349)\end{array}$ & $P$ value & SD & $\begin{array}{l}\text { Pre-PCI TIMI 0/1 } \\
(\mathrm{n}=2473)\end{array}$ & $\begin{array}{l}\text { Pre-PCI TIMI } 2 / 3 \\
(n=2473)\end{array}$ & $P$ value & SD \\
\hline \multicolumn{9}{|l|}{ IRA } \\
\hline Left main, n (\%) & $40(1.3)$ & $166(3.8)$ & $<.001$ & -1.59 & $40(1.6)$ & $32(1.3)$ & .406 & 0.16 \\
\hline LAD, n (\%) & $1112(35.2)$ & $2054(47.2)$ & $<.001$ & -3.30 & $996(40.3)$ & $1005(40.6)$ & .817 & -0.08 \\
\hline LCx, n (\%) & $970(30.7)$ & $1024(23.5)$ & $<.001$ & 2.04 & $698(28.2)$ & $685(27.7)$ & .704 & 0.14 \\
\hline RCA, n (\%) & 1035 (32.8) & $1105(25.4)$ & $<.001$ & 2.06 & 739 (29.9) & $751(30.4)$ & .733 & -0.14 \\
\hline $\mathrm{LAD}, \mathrm{n}(\%)$ & $1,559(49.4)$ & $2644(60.8)$ & $<.001$ & -3.01 & $1338(54.1)$ & $1345(54.4)$ & .842 & -0.08 \\
\hline LCX, n (\%) & $1318(41.7)$ & $1608(37.0)$ & $<.001$ & 1.26 & $987(39.9)$ & $976(39.5)$ & .771 & 0.11 \\
\hline RCA, n (\%) & $1284(40.7)$ & $1497(34.4)$ & $<.001$ & 1.69 & 959 (38.3) & 966 (39.1) & .861 & -0.21 \\
\hline \multicolumn{9}{|l|}{ Extent of CAD } \\
\hline Single-vessel disease, n (\%) & $1402(44.4)$ & $1978(45.5)$ & .357 & -0.29 & $1104(44.6)$ & $1101(44.5)$ & .932 & 0.03 \\
\hline Two-vessel disease, $n(\%)$ & 1066 (33.8) & 1475 (33.9) & .892 & -0.03 & 847 (34.2) & 839 (33.9) & .834 & 0.08 \\
\hline Type C, n (\%) & $1558(49.4)$ & $1501(34.5)$ & $<.001$ & 3.94 & $1087(44.0)$ & $1114(45.0)$ & .457 & -0.27 \\
\hline In-hospital GP IIb/lla inhibitor & $374(11.8)$ & $278(6.4)$ & $<.001$ & 2.04 & $219(8.9)$ & $219(8.9)$ & 1.000 & 0 \\
\hline IVUS, n (\%) & $579(18.3)$ & $1077(24.8)$ & $<.001$ & -2.13 & $504(20.4)$ & 485 (19.6) & .500 & 0.25 \\
\hline OCT, n (\%) & $23(0.7)$ & $68(1.6)$ & .001 & -0.61 & $22(0.9)$ & $25(1.0)$ & .664 & -0.07 \\
\hline FFR, n (\%) & $18(0.6)$ & $96(2.2)$ & $<.001$ & -1.10 & $18(0.7)$ & $17(0.7)$ & .865 & 0.03 \\
\hline $\mathrm{PCl}$ within $24 \mathrm{hr}$ & 2729 (86.4) & 3669 (84.4) & $<.001$ & 0.72 & 2108 (85.2) & $2101(85.0)$ & .780 & 0.07 \\
\hline \multicolumn{9}{|l|}{ Drug-eluting stents } \\
\hline ZES, n (\%) & 1131 (35.8) & $1383(31.8)$ & $<.001$ & 1.10 & $844(34.1)$ & 860 (34.8) & .654 & -0.20 \\
\hline EES, n (\%) & 1616 (51.2) & $2210(50.8)$ & .751 & 0.11 & $1284(51.9)$ & $1270(51.4)$ & .690 & 0.13 \\
\hline BES, n (\%) & 437 (13.8) & 762 (17.5) & $<.001$ & -1.33 & 366 (14.8) & 364 (14.7) & .936 & 0.04 \\
\hline Others, n (\%) ${ }^{*}$ & $44(1.4)$ & $97(2.2)$ & .010 & -0.50 & $40(1.6)$ & $40(1.6)$ & 1.000 & 0 \\
\hline Multivessel PCl & $1537(48.7)$ & $2088(48.0)$ & .564 & 0.18 & 1217 (49.2) & 1195 (48.3) & .550 & 0.24 \\
\hline
\end{tabular}

Values are means $\pm \mathrm{SD}$ or numbers and percentages. The $P$ values for continuous data were obtained from the unpaired $t$ test. The $P$ values for categorical data from chi-square or Fisher exact test. ACC/AHA $=$ American College of Cardiology/American Heart Association, $\mathrm{BES}=$ biolimus-eluting stents, $\mathrm{CAD}=$ coronary artery disease, $\mathrm{EES}=$ everolimus-eluting stent, $\mathrm{FFR}=\mathrm{fractional}$ flow reserve, GP=glycoprotein, IRA = infarct-related artery, IVUS = intravascular ultrasound, $L A D=$ left anterior descending coronary artery, $L C x=$ left circumflex coronary artery, OCT=optical coherence tomography, Pre-PCl= pre-percutaneous coronary intervention, PSM = propensity-score matched, RCA = right coronary artery, SD = standardized mean difference, TIMI=thrombolysis in myocardial infarction, ZES = zotarolimus-eluting stent

* Others mean that other 2G-DES excluding ZES, EES, and BES.

2 years, the cumulative incidences of re-MI, any repeat revascularization, and ST were similar between the 2 groups. Third, old age, hypertension, DM, previous PCI, decreased LVEF, cardiogenic shock, CPR on admission, angiotensinconverting enzyme inhibitors, lipid-lowering agents, multivessel disease, and the occurrence of ventricular tachycardia (VT)/ ventricular fibrillation (VF) were independent predictors of both all-cause death and CD.

The previous report suggested that pre-procedural TIMI flow grade 3 was a stronger prognostic predictor than post-procedural TIMI flow grade $3{ }^{[3]}$ In a study by Bailleul et al, ${ }^{[5]}$ the risk of death after adjustment was similar between the pre-TIMI $0 / 1$ and pre-TIMI 2/3 groups. Although the follow-up duration of their study ${ }^{[5]}$ was longer than our study, the total number of enrolled patients was relatively smaller than this study, and the number of patients who received DES was $<45 \%$ in the total enrolled NSTEMI patients. Therefore, their results could be different from those of our study. Our results may be consistent with previous reports $^{[2-4]}$ that showed significantly decreased mortality of the pre-TIMI 2/3 group in patients with STEMI.
Previous studies ${ }^{[15,16]}$ showed spontaneous reperfusions before definitive PCI would be expected to improve outcomes by enhancing myocardial salvage, which preserves ventricular function. Therefore, those patients achieving TIMI-3 flow before PCI would be expected to present with less heart failure and demonstrate greater preservation of regional and global LVEF and have improved early and late survival. ${ }^{[3]}$ Other advantages include improved technical success from the initial lesion delineation, road mapping, ${ }^{[3]}$ and fewer catheterization laboratory events. ${ }^{[2]}$ Hashimoto et $\mathrm{al}^{[7]}$ suggested that high systolic blood pressure (SBP) was significantly associated with pre-TIMI 3, and SBP might increase because of myocardial reperfusion. ${ }^{[17]}$ In our study, mean SBP was significantly higher in the pre-TIMI $2 / 3$ group than in the pre-TIMI $0 / 1$ group $(133.7 \pm 26.4 \mathrm{mmHg}$ vs $135.5 \pm 25.9$ $\mathrm{mmHg}, P=.002)$. Additionally, consistent with the Hashimoto et $\mathrm{al}^{[7]}$ study, the cumulative incidence of VT/VF was significantly higher in the pre-TIMI $0 / 1$ than in the pre-TIMI $2 / 3(52 / 3157$ [1.6\%] vs $38 / 4349$ [0.9\%], $P=.003)$ during the 2 -year follow-up period. 
Table 3

Clinical outcomes.

\begin{tabular}{|c|c|c|c|c|c|}
\hline \multirow[b]{2}{*}{ Outcomes } & \multicolumn{5}{|c|}{ Cumulative events (\%) } \\
\hline & Pre-PCI TIMI 0/1 & Pre-PCI TIMI 2/3 & Log-rank & Hazard ratio $(95 \% \mathrm{Cl})$ & $P$ value \\
\hline \multicolumn{6}{|l|}{30 days } \\
\hline MACES & $97(3.1)$ & $82(1.9)$ & .001 & 1.642 (1.224-2.203) & .001 \\
\hline All-cause death & $74(2.3)$ & $54(1.2)$ & $<.001$ & 1.902 (1.339-2.701) & $<.001$ \\
\hline Cardiac death & $69(2.2)$ & $45(1.0)$ & $<.001$ & 2.127 (1.461-3.097) & $<.001$ \\
\hline Re-Ml & $18(0.6)$ & $22(0.5)$ & .686 & $1.137(0.610-2.120)$ & .686 \\
\hline ST (probable or definite) & $8(0.3)$ & $10(0.2)$ & .838 & $1.102(0.435-2.792)$ & .838 \\
\hline \multicolumn{6}{|l|}{ PSM patients } \\
\hline MACES & $68(2.8)$ & $45(1.8)$ & .028 & 1.519 (1.042-2.214) & .030 \\
\hline All-cause death & $49(2.0)$ & $28(1.1)$ & .016 & 1.759 (1.106-2.798) & .017 \\
\hline Cardiac death & $47(1.9)$ & $25(1.0)$ & .009 & 1.889 (1.163-3.069) & .010 \\
\hline Re-Ml & $14(0.6)$ & $11(0.4)$ & .537 & 1.281 (0.582-2.822) & .539 \\
\hline \multicolumn{6}{|l|}{ Total study population } \\
\hline MACES & 303 (10.2) & $355(9.1)$ & .068 & 1.153 (0.989-1.344) & .068 \\
\hline All-cause death & $155(5.1)$ & $158(4.0)$ & .010 & 1.335 (1.070-1.667) & .011 \\
\hline Cardiac death & $111(3.7)$ & $102(2.6)$ & .004 & $1.486(1.136-1.945)$ & .004 \\
\hline Re-Ml & $64(2.3)$ & $81(2.2)$ & .697 & 1.067 (0.769-1.481) & .697 \\
\hline All-cause death or Ml & $211(7.1)$ & $227(5.9)$ & .014 & $1.263(1.047-1.524)$ & .014 \\
\hline Any repeat revascularization & $115(4.1)$ & $168(4.5)$ & .463 & $1.093(0.862-1.376)$ & .463 \\
\hline ST (probable or definite) & $19(0.6)$ & $22(0.5)$ & .577 & 1.190 (0.644-2.199) & .578 \\
\hline \multicolumn{6}{|l|}{ PSM patients } \\
\hline MACES & $234(10.1)$ & $179(8.0)$ & .009 & $1.294(1.065-1.572)$ & .009 \\
\hline All-cause death & $116(4.9)$ & $74(3.3)$ & .003 & $1.559(1.165-2.087)$ & .003 \\
\hline Cardiac death & $84(3.5)$ & $51(2.2)$ & .005 & 1.641 (1.159-2.325) & .005 \\
\hline
\end{tabular}

MACES = major adverse cardiac events, Pre-PCl= pre-percutaneous coronary intervention, $\mathrm{PSM}=$ propensity-score matched, $\mathrm{Re}-\mathrm{Ml}=$ recurrent myocardial infarction, $\mathrm{ST}=$ stent thrombosis, $\mathrm{TIMI}=$ thrombolysis in myocardial infarction.

In our study, during the first 1 month, MACE, all-cause death, $\mathrm{CD}$, and all-cause death or MI were significantly higher in the pre-TIMI 0/1 than in the pre-TIMI 2/3 (Table 3). In the Brodie et $\mathrm{al}^{[2]}$ study, 30 days mortality rate of the STEMI patients was better in the pre-TIMI $2 / 3$ group than in the pre-TIMI 0/1 group (4.8\% vs $8.9 \%, P=.02$ ). In the Rakowski et al study, ${ }^{[4]} 30$ days all-cause death rate of the STEMI patients was significantly lower in the pre-TIMI $2 / 3$ group than in the pre-TIMI $0 / 1$ group (relative risk [RR]: 2.38; 95\% CI: 1.27-4.47; $P=.005$ ). Therefore, our results revealed that the mortality reduction benefit of pre-PCI patency of IRA in patients with NSTEMI might be similar to those results in patients with STEMI. As mentioned (Table S1, Supplemental Digital Content, http://links.lww.com/ MD/G364), old age, hypertension, DM, and previous PCI were meaningful independent predictors of both all-cause death and CD in this study. In Table 1 , the numbers of those patients were significantly higher in the pre-TIMI $2 / 3$ group. Despite these poor baseline characteristics in the pre-TIMI 2/3 group, the 2-year cumulative major clinical outcomes, including MACEs, all-cause death, CD, and all-cause death or MI, were better in the pre-TIMI $2 / 3$ group than in the pre-TIMI 0/1 group. Hence, our results seem to show the important role of pre-PCI coronary flow grade in determining long-term mortality in addition to significant independent predictors. Karwowski et $\mathrm{al}^{[18]}$ mentioned that although there is a lack of data in patients with NSTEMI and total occlusion, probably, in the case of complete interruption of blood supply, rapid restoration of flow could result in smaller infarct size and better prognosis. More recently, Kim et al ${ }^{[19]}$ showed that an early invasive strategy ( $\mathrm{PCI} \leq 24$ hours) is preferred to a delayed invasive strategy in reducing all-cause death in patients with pre-TIMI 0/1. However, in patients with pre-TIMI 2/3, the clinical endpoint was similar between the 2 strategies. Based on these results ${ }^{[18,19]}$ we assume that pre-TIMI after successful PCI could be useful to select the next step treatment strategy in patients with NSTEMI.

To date, the effect of pre-TIMI $2 / 3$ in patients with NSTEMI was not fully illuminated. More than 50 community and teaching hospitals in South Korea participated in this nationwide retrospective observational multicenter registry analysis. Hence, our study could provide significant information about the comparative benefit of pre-PCI patent IRA for reducing mortality rate compared with lack of patency in patients with NSTEMI.

This study had several limitations. First, because this study was a retrospective cohort study, some data might have been 

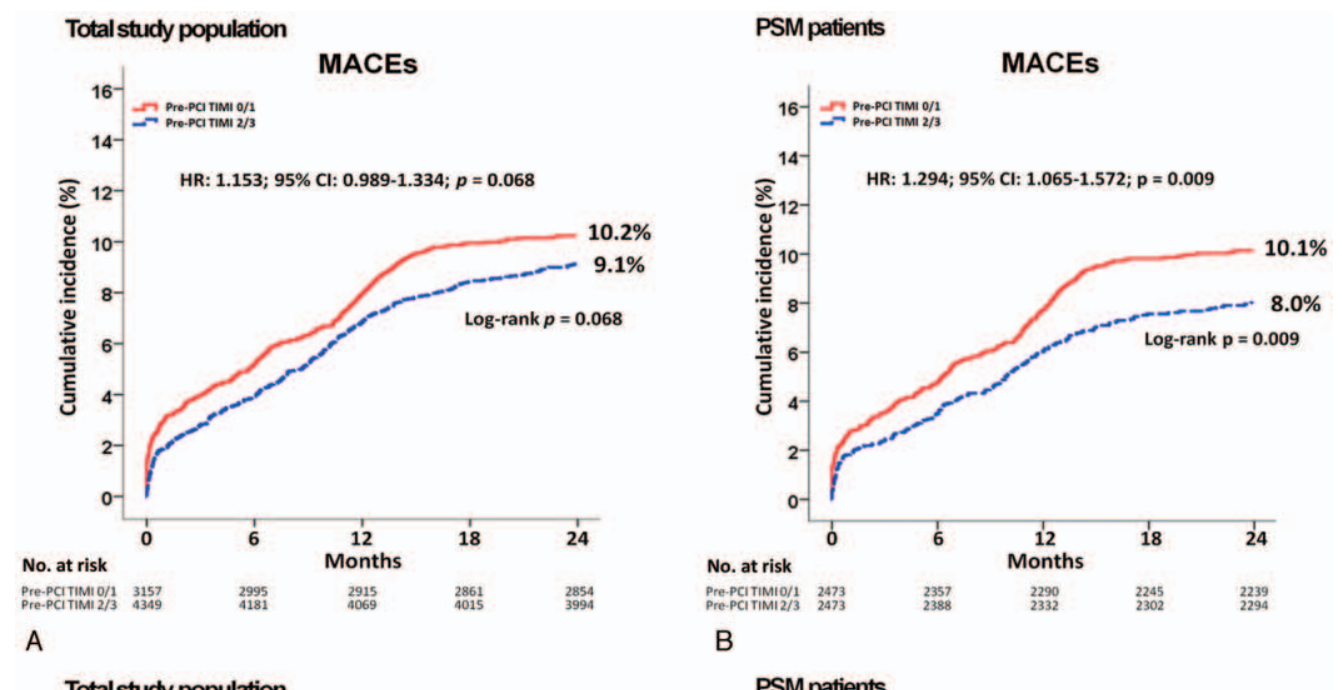

PSM patients

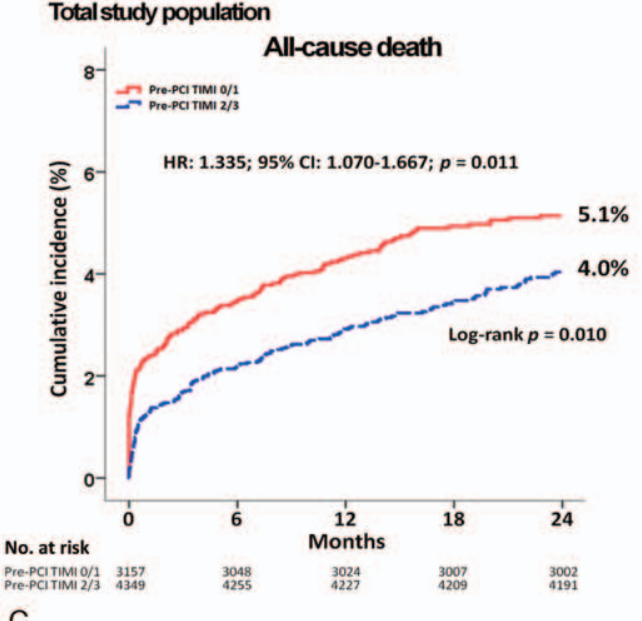

C

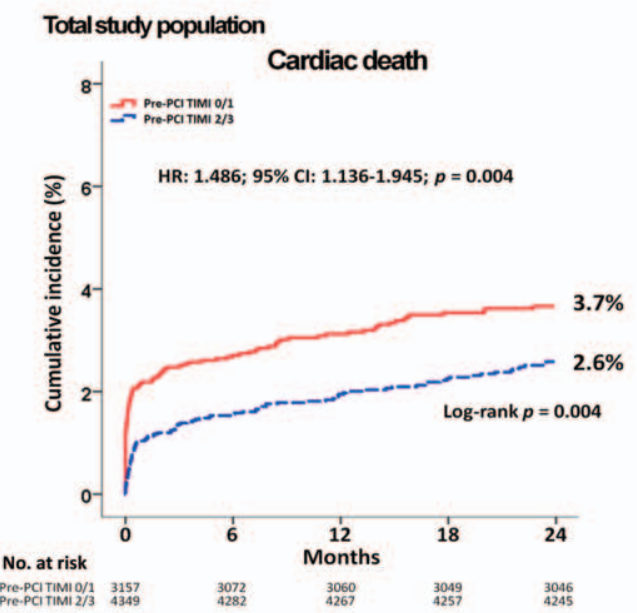

$\mathrm{E}$

Figure 2. Kaplan-Meier analysis for major adverse cardiac events (MACEs; $A$ and B), all-cause death (C and D), cardiac death (E and F), recurrent myocardial infarction (Re-Ml; $\mathrm{G}$ and $\mathrm{H}$ ), all-cause death or $\mathrm{Ml}(\mathrm{I}$ and $\mathrm{J}$ ), any repeat revascularization (K and L), and stent thrombosis ( $\mathrm{M}$ and $\mathrm{N}$ ) in the total study population (A, C, $\mathrm{E}, \mathrm{G}, \mathrm{I}, \mathrm{K}$, and $\mathrm{M})$, and propensity-score matched (PSM) patients (B, D, F, H, J, L, and N) at 2 years. $\mathrm{Cl}=$ confidence interval, $\mathrm{HR}=$ hazard ratio, Pre-PCl= prepercutaneous coronary intervention.

underreported and/or missed. Second, the 2-year follow-up duration was insufficient to evaluate the long-term adverse events. Third, ACEIs and lipid-lowering agents were meaningful independent predictors of all-cause death and CD. However, because this study was based on discharge medications, we could not precisely determine the adherence or non-adherence of the 

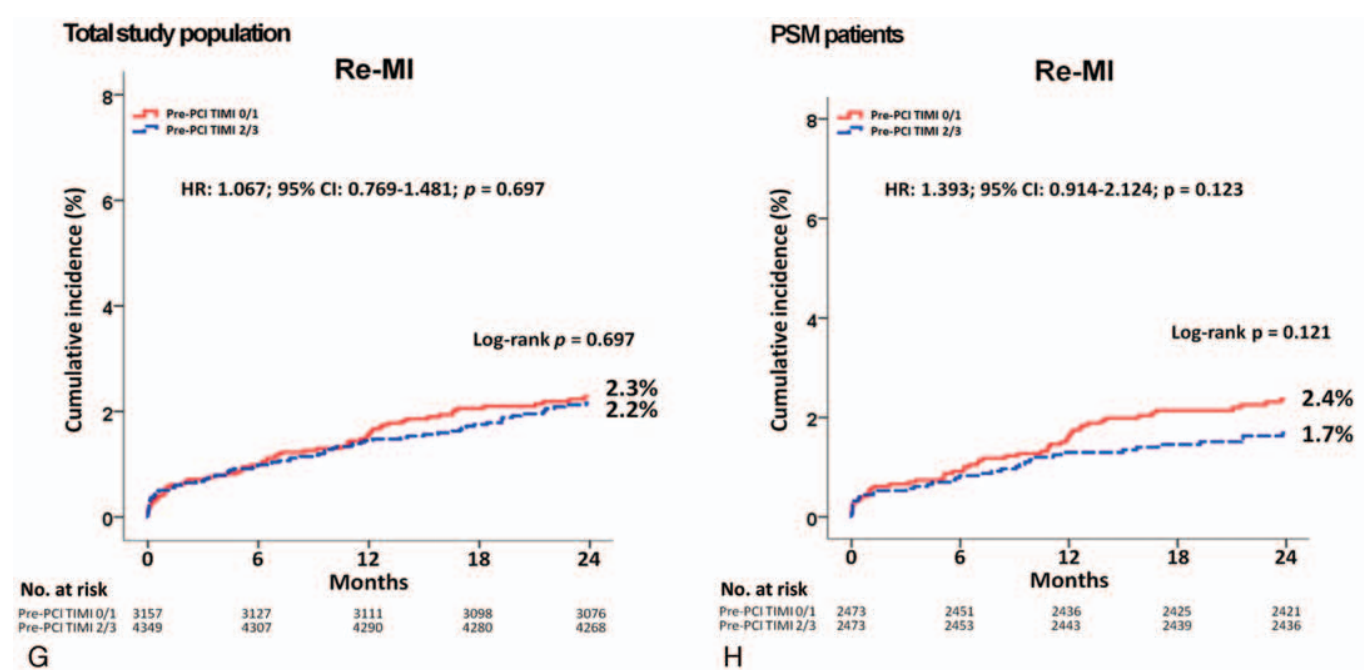

\section{Total study population}

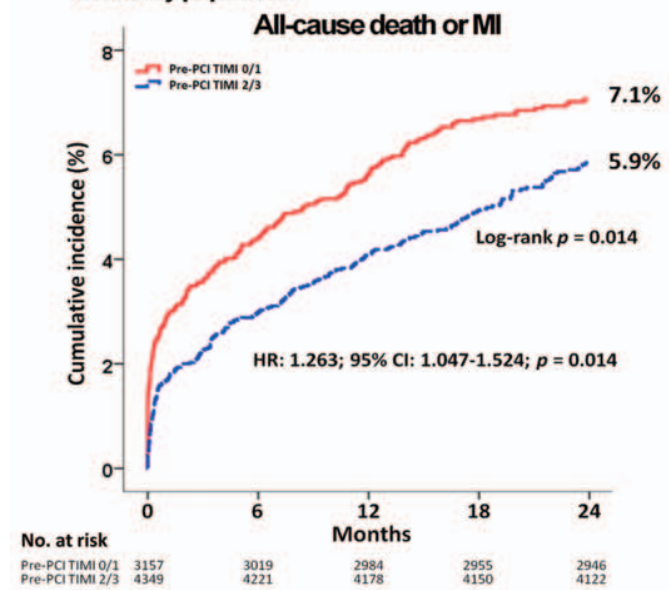

$\mathrm{H}$

PSM patients

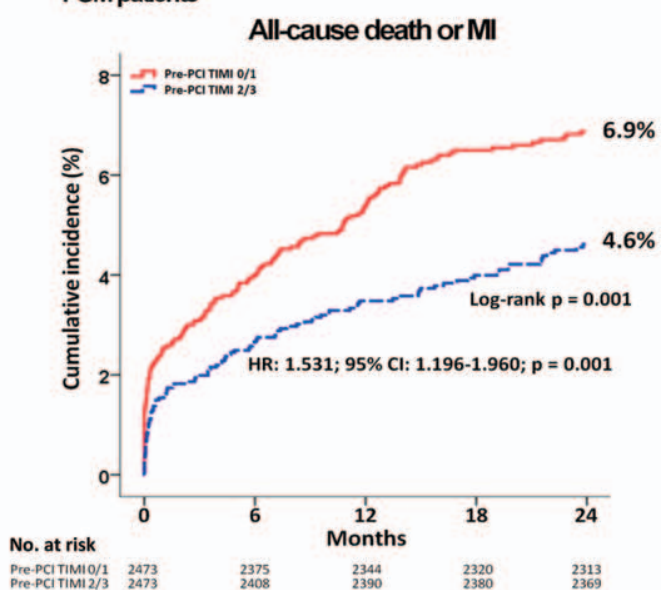

I

Total study population

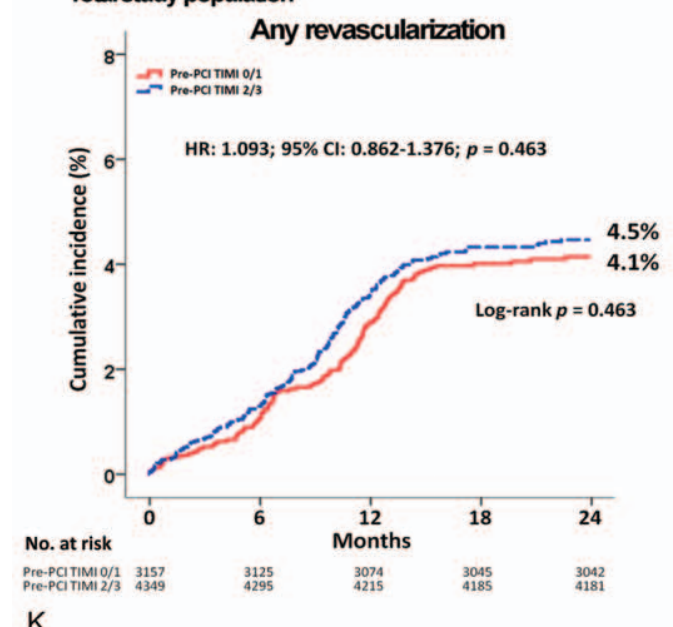

J

PSM patients

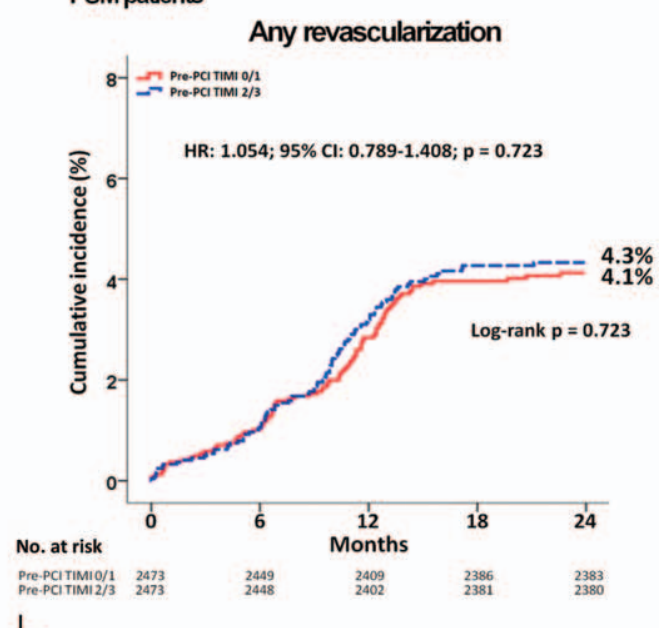

Figure 2. (Continued)

enrolled patients to their prescribed discharge medications during the follow-up period. Fourth, although PSM analysis was performed in this study, variables not included in the KAMIR may have affected the study outcomes. Finally, infarction size is more accurately correlated with mortality following an AMI rather than the flow grade of the IRA. ${ }^{[11]}$ Moreover, various strategies can be used to measure the infarct size including technetium-99m sestamibi single-photon emission computed tomography myocardial perfusion imaging and cardiac magnetic resonance imaging. ${ }^{[20]}$ However, because these optimized diag- 

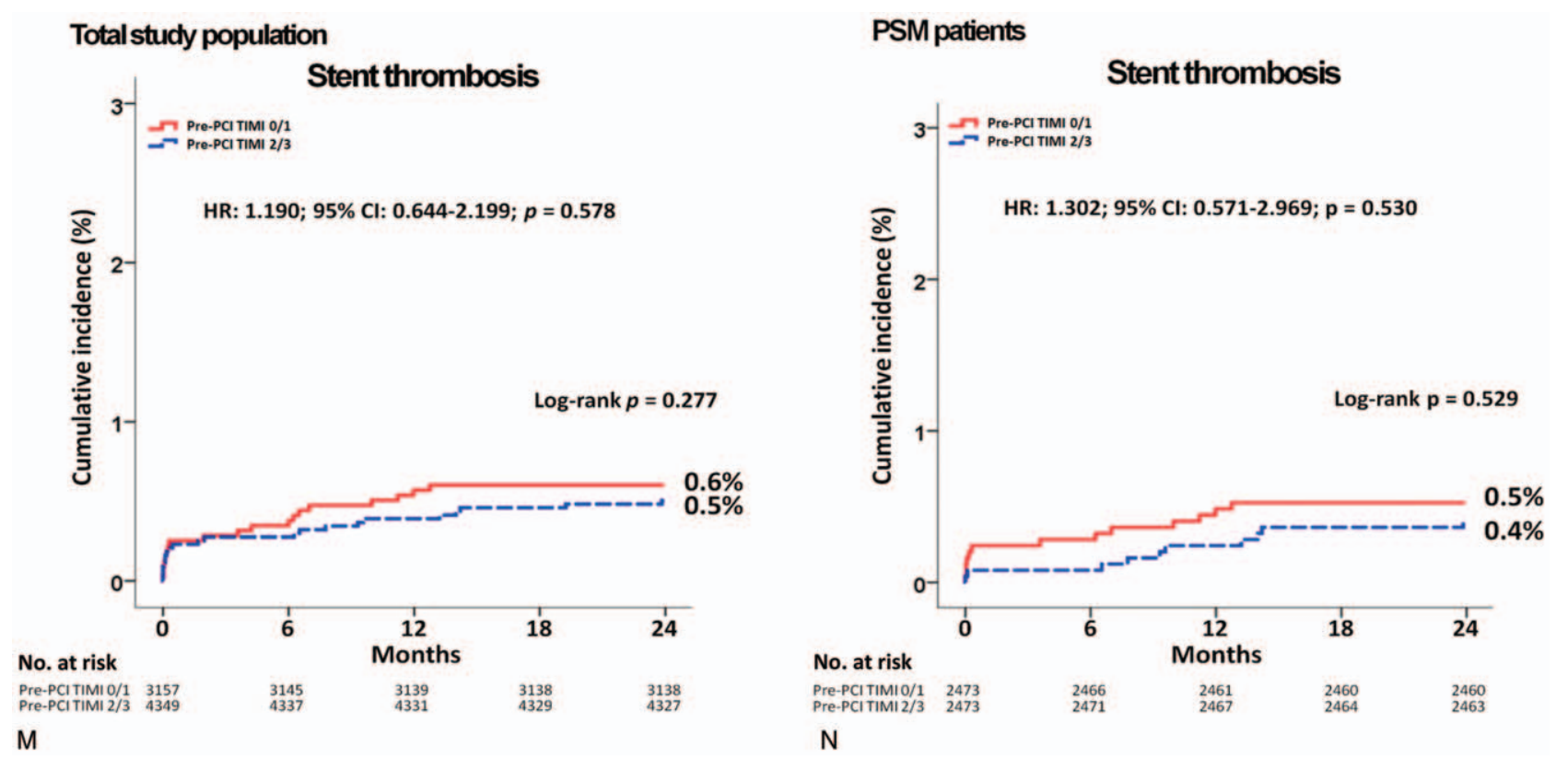

Figure 2. (Continued)

nostic tools were not mandatory in the KAMIR data, we could not provide this information concerning the size of myocardial infarction. Therefore, this is a major shortcoming of this study.

In conclusion, among a contemporary cohort of NSTEMI, these data suggest that the presence of pre-PCI patency of the infarct-related artery showed better clinical outcomes than those with a lack of patency concerning mortality. However, further studies are warranted to elucidate this focus.

\section{Acknowledgments}

The authors thank all of the clinical investigators who contributed time and effort to this study, as well as the Korea Acute Myocardial Infarction (KAMIR) Investigators.

Korea Acute Myocardial infarction Registry (KAMIR) investigators.

Myung Ho Jeong, MD, Youngkeun Ahn, MD, Sung Chul Chae, MD, Jong Hyun Kim, MD, Seung-Ho Hur, MD, Young Jo Kim, MD, In Whan Seong, MD, Donghoon Choi, MD, Jei Keon Chae, MD, Taek Jong Hong, MD, Jae Young Rhew, MD, Doo-Il Kim, MD, In-Ho Chae, MD, Junghan Yoon, MD, Bon-Kwon Koo, MD, Byung-Ok Kim, MD, Myoung Yong Lee, MD, Kee-Sik Kim, MD, Jin-Yong Hwang, MD, Myeong Chan Cho, MD, Seok Kyu Oh, MD, Nae-Hee Lee, MD, Kyoung Tae Jeong, MD, Seung-Jea Tahk, MD, Jang-Ho Bae, MD, Seung-Woon Rha, MD, Keum-Soo Park, MD, Chong Jin Kim, MD, Kyoo-Rok Han, MD, Tae Hoon Ahn, MD, Moo-Hyun Kim, MD, Ki Bae Seung, MD, Wook Sung Chung, MD, Ju-Young Yang, MD, Chong Yun Rhim, MD, Hyeon-Cheol Gwon, MD, Seong-Wook Park, MD, Young-Youp Koh, MD, Seung Jae Joo, MD, Soo-Joong Kim, MD, Dong Kyu Jin, MD, Jin Man Cho, MD, Sang-Wook Kim, MD, Jeong Kyung Kim, MD, Tae Ik Kim, MD, Deug Young Nah, MD, Si Hoon Park, MD, Sang Hyun Lee, MD, Seung Uk Lee, MD, Hang-Jae Chung, MD, Jang-Hyun Cho, MD, Seung Won Jin, MD, Myeong-Ki Hong, MD, Yangsoo Jang, MD, Jeong Gwan Cho, MD, Hyo-Soo Kim, MD and Seung-Jung Park, MD.

\section{Author contributions}

Conceptualization: Yong Hoon Kim, Ae-Young Her, Myung Ho Jeong, Byeong-Keuk Kim, Sung-Jin Hong, Seunghwan Kim, Chul-Min Ahn, Jung-Sun Kim, Young-Guk Ko, Donghoon Choi, Myeong-Ki Hong, Yangsoo Jang.

Data curation: Yong Hoon Kim, Ae-Young Her, Sung-Jin Hong, Seunghwan Kim, Jung-Sun Kim, Myeong-Ki Hong, Yangsoo Jang.

Formal analysis: Yong Hoon Kim, Ae-Young Her, Sung-Jin Hong, Seunghwan Kim.

Funding acquisition: Myung Ho Jeong.

Investigation: Yong Hoon Kim, Ae-Young Her, Myung Ho Jeong, Byeong-Keuk Kim, Sung-Jin Hong, Seunghwan Kim, Chul-Min Ahn, Jung-Sun Kim, Young-Guk Ko, Donghoon Choi, Myeong-Ki Hong, Yangsoo Jang.

Methodology: Yong Hoon Kim, Ae-Young Her, Myung Ho Jeong, Byeong-Keuk Kim, Sung-Jin Hong, Seunghwan Kim, Chul-Min Ahn, Jung-Sun Kim, Young-Guk Ko, Donghoon Choi, Myeong-Ki Hong, Yangsoo Jang.

Project administration: Yong Hoon Kim, Ae-Young Her, Myung Ho Jeong, Byeong-Keuk Kim, Sung-Jin Hong, Seunghwan Kim, Chul-Min Ahn, Jung-Sun Kim, Young-Guk Ko, Donghoon Choi, Myeong-Ki Hong, Yangsoo Jang.

Resources: Myung Ho Jeong, Byeong-Keuk Kim, Sung-Jin Hong, Seunghwan Kim, Chul-Min Ahn, Jung-Sun Kim, Young-Guk Ko, Donghoon Choi, Myeong-Ki Hong, Yangsoo Jang.

Software: Yong Hoon Kim, Ae-Young Her, Sung-Jin Hong, Seunghwan Kim, Jung-Sun Kim, Myeong-Ki Hong.

Supervision: Yong Hoon Kim, Myung Ho Jeong, Donghoon Choi, Myeong-Ki Hong, Yangsoo Jang.

Validation: Yong Hoon Kim, Ae-Young Her, Myung Ho Jeong, Byeong-Keuk Kim, Sung-Jin Hong, Seunghwan Kim, ChulMin Ahn, Jung-Sun Kim, Young-Guk Ko, Donghoon Choi, Myeong-Ki Hong, Yangsoo Jang. 
Visualization: Yong Hoon Kim, Ae-Young Her, Myung Ho Jeong, Byeong-Keuk Kim, Sung-Jin Hong, Seunghwan Kim, Chul-Min Ahn, Jung-Sun Kim, Young-Guk Ko, Donghoon Choi, Myeong-Ki Hong, Yangsoo Jang.

Writing - original draft: Yong Hoon Kim, Ae-Young Her.

Writing - review \& editing: Yong Hoon Kim, Ae-Young Her, Myeong-Ki Hong, Yangsoo Jang.

\section{References}

[1] Guo Z, Yang X. Does pre-angiography Total ST-segment resolution reliably predict spontaneous reperfusion of the infarct-related artery in patients with acute myocardial infarction? BMC Cardiovasc Disord 2019;19:264.

[2] Brodie BR, Stuckey TD, Hansen C, et al. Benefit of coronary reperfusion before intervention on outcomes after primary angioplasty for acute myocardial infarction. Am J Cardiol 2000;85:13-8.

[3] Stone GW, Cox D, Garcia E, et al. Normal flow (TIMI-3) before mechanical reperfusion therapy is an independent determinant of survival in acute myocardial infarction: analysis from the primary angioplasty in myocardial infarction trials. Circulation 2001;104: 636-41.

[4] Rakowski T, Dudek D, Dziewierz A, et al. Impact of infarct-related artery patency before primary PCI on outcome in patients with ST-segment elevation myocardial infarction: the HORIZONS-AMI trial. EuroIntervention 2013;8:1307-14.

[5] Bailleul C, Aissaoui N, Cayla G, et al. FAST-MI investigators. Prognostic impact of prepercutaneous coronary intervention TIMI flow in patients with ST-segment and non-ST-segment elevation myocardial infarction: results from the FAST-MI 2010 registry. Arch Cardiovasc Dis 2018; 111:101-8.

[6] De Luca G, Brener SJ, Mehran R, et al. Implications of pre-procedural TIMI flow in patients with non ST-segment elevation acute coronary syndromes undergoing percutaneous coronary revascularization: insights from the ACUITY trial. Int J Cardiol 2013;167:727-32.

[7] Hashimoto T, Ako J, Nakao K, et al. J-MINUET investigators. Preprocedural thrombolysis in myocardial infarction flow in patients with st-segment elevation myocardial infarction. Int Heart J 2018;59:920-5.

[8] Sarkar A, Lee JJ. TIMI Grade Flow, StatPearls, StatPearls Publishing Copyright (C) 2020. Treasure Island, FL: StatPearls Publishing LLC; 2020.

[9] Kim JH, Chae SC, Oh DJ, et al. Korea acute myocardial infarctionnational institutes of health registry investigators. Multicenter cohort study of acute myocardial infarction in korea - interim analysis of the
Korea Acute Myocardial Infarction Registry-National Institutes of Health Registry. Circ J 2016;80:1427-36.

[10] Grech ED. ABC of interventional cardiology: percutaneous coronary intervention. II: the procedure. BMJ 2003;326:1137-40.

[11] Amsterdam EA, Wenger NK, Brindis RG, et al. 2014AHA/ACC guideline for the management of patients with non-st-elevation acute coronary syndromes: a report of the american College of Cardiology/ American Heart Association Task Force on Practice Guidelines. J Am Coll Cardiol 2014;64:e139-228.

[12] Newby LK, Jesse RL, Babb JD, et al. ACCF 2012 expert consensus document on practical clinical considerations in the interpretation of troponin elevations: a report of the American College of Cardiology Foundation task force on Clinical Expert Consensus Documents. J Am Coll Cardiol 2012;60:2427-63.

[13] TIMI Study Group. The Thrombolysis in Myocardial Infarction (TIMI) trial. Phase I findings. N Engl J Med 1985;312:932-6.

[14] Kim YH, Her AY, Jeong MH, et al. Impact of renin-angiotensin system inhibitors on long-term clinical outcomes in patients with acute myocardial infarction treated with successful percutaneous coronary intervention with drug-eluting stents: Comparison between STEMI and NSTEMI. Atherosclerosis 2019;280:166-73.

[15] Lamas GA, Flaker GC, Mitchell G, et al. and for the Survival Ventricular Enlargement Investigators. Effect of infarct artery patency on prognosis after acute myocardial infarction. The Survival and Ventricular Enlargement Investigators. Circulation 1995;92:1101-9.

[16] Braunwald E. The open-artery theory is alive and well-again. N Engl J Med 1993;329:1650-2.

[17] Shiraishi J, Nakamura T, Shikuma A, et al. AMI-Kyoto Multi-Center Risk Study Group. Relationship between mean blood pressure at admission and in-hospital outcome after primary percutaneous coronary intervention for acute myocardial infarction. Int Heart J 2016;57: $547-52$.

[18] Karwowski J, Poloński L, Gierlotka M, et al. Total coronary occlusion of infarct-related arteries in patients with non-ST-elevation myocardial infarction undergoing percutaneous coronary revascularisation. Kardiol Pol 2017;75:108-16.

[19] Kim YH, Her AY, Jeong MH, et al. Two-year clinical outcomes according to Pre-PCI TIMI flow grade and reperfusion timing in NonSTEMI after newer-generation drug-eluting stents implantation. Angiology 2021;33197211012537doi: 10.1177/00033197211012537. Online ahead of print.

[20] Carlsson M, Ubachs JF, Hedström E, et al. Myocardium at risk after acute infarction in humans on cardiac magnetic resonance: quantitative assessment during follow-up and validation with singlephoton emission computed tomography. JACC Cardiovasc Imaging 2009;2:569-76. 